\title{
Myasthenia Gravis Related to Thymic Carcinoma: A Case Study
}

\author{
Daniel Thomas Pereira Lopes ${ }^{10}$ Samila Marissa Pinheiro Gomes ${ }^{1}$ Sheila Aparecida Coelho Siqueira ${ }^{2}$ \\ Fernando Pereira Frassetto ${ }^{2}$ Pedro Henrique Martins Arruda Sampaio ${ }^{1}$ \\ Cristiane Araujo Martins Moreno ${ }^{1,3}$ Maria Sheila Guimarães Rocha ${ }^{1,4}$ Eduardo Paula Estephan ${ }^{1,5}$
}

${ }^{1}$ Neurology Departament, Hospital Santa Marcelina, Sao Paulo, São Paulo, Brazil

2 Pathology Departament, Universidade de Sao Paulo Hospital das Clinicas, Sao Paulo, São Paulo, Brazil

${ }^{3}$ Neurology Departament, Universidade de Sao Paulo Hospital das Clinicas, Sao Paulo, São Paulo, Brazil

${ }^{4}$ Neurology Departament, Faculdade Santa Marcelina Curso de Medicina, Sao Paulo, Sao Paulo, Brazil

${ }^{5}$ Neurology Departament, Faculdade de Medicina da Universidade de São Paulo, Sao Paulo, São Paulo, Brazil

Thorac Cardiovasc Surg Rep 2020;9:e33-e36.
Address for correspondence Daniel Thomas Pereira Lopes, MD, Hospital Santa Marcelina, Santa Marcelina Street, Sao Paulo 08270-070, Brazil (e-mail: sopelinad@gmail.com; dt-lopes@uol.com.br).

\begin{abstract}
Keywords

- thymus

- neurology/neurologic

- thoracic surgery

- pathology

Myasthenia gravis and thymoma are often presented in association with $\sim 10 \%$ of myasthenic cases having concomitant thymoma. Thymic carcinoma is one of the rarest/aggressive human epithelial tumors and has no correlation with myasthenia gravis hitherto. Here is provided a clinical case and review of literature on a very rare association of thymic carcinoma (with no sign of thymoma) and myasthenia gravis (antiacetylcholine receptor antibody positive). Two years after thymectomy, clinical evolution was satisfactory. This clinical case elicits hypothesis that thymic carcinoma may be related with myasthenia gravis, what may have good prognostic from oncologic and neurologic perspectives.
\end{abstract}

\section{Introduction}

Myasthenia gravis (MG) is an autoimmune disease with an annual incidence of 1.7 to 21.3 per million. ${ }^{1}$ It is characterized by functional abnormalities caused by autoantibodies attacking components of the neuromuscular junction, ${ }^{2}$ a highly specialized synapse between a motor axon and a muscle fiber that contains specific protein complexes necessary for maximum efficacy on the stimulus transmission. Due to its complexity and lack of blood barrier, this structure is vulnerable to genetic disorders ${ }^{3,4}$ and immunologic attack. ${ }^{2,5}$

The clinical hallmark of MG is fatigability and fluctuating muscle weakness, most often affecting ocular muscles, with or without other muscles. However, clinical features are highly variable, even between patients expressing the same antibody, which can make diagnosis difficult ${ }^{6,7}$ Antibodies most commonly target the acetylcholine receptor (AChR), but in 50\% of patients with ocular MG, and 10 to $15 \%$ with generalized disease, tests detecting anti-AChR antibodies are negative. ${ }^{2}$ These seronegative cases are often associated with antibodies against muscle-specific receptor tyrosine kinase or low-density lipoprotein receptor-related protein $4,{ }^{2}$ but a few cases are seronegative for all three antibodies (sometimes called triple seronegative MG).

The origin of autoimmunity can be related to thymic abnormalities, defects in immune regulation and hormones, with a combination of predisposing factors (genetic susceptibility, dysregulation of microRNAs, impact of sex hormones on immune cells) and environmental factors (such as drugs and pathogens). ${ }^{2}$ of the thymic abnormalities, the relationship received

March 12, 2020 accepted after revision May 6, 2020
DOI https://doi.org/

10.1055/s-0040-1714075. ISSN 2194-7635.
๑) 2020 Georg Thieme Verlag KG
Stuttgart · New York

License terms

(๑) $\Theta \circledast$ 
between thymoma and anti-AChR MG is well known. Although the role of autoimmunity in thymoma is not totally clear, it is known that thymic cells exported to the periphery may include undeleted autoreactive T cells with deficient molecular components important for tolerance. ${ }^{5}$

Thymic carcinoma (TC), however, is a malignant epithelial tumor with cytologic atypia, without any thymus-like features; this is one reason why it is not, in theory, expected to accompany MG. ${ }^{8}$ TC is among the rarest human neoplasms (constituting only $5 \%$ of all thymic neoplasms ${ }^{9}$ ), and is characterized by its aggressiveness and poor prognosis (5-year survival rate, $15-65 \%{ }^{10}$ ), due to its invasiveness and metastasizing potential. Histological features of this tumor are anaplasia, cell atypia, paucity of lymphocytes and increased proliferation, enlarged nuclei, coarse chromatin, discrete macronucleoli and a moderate amount of cytoplasm. ${ }^{9}$

A large retrospective Japanese analysis estimated a rate of MG of $1 \%$ in patients with TC. ${ }^{11}$ Generally, in such cases, at least some thymoma is observed within the carcinoma. ${ }^{12}$ However, we observed a case of MG related to a circumscribed TC, with no thymoma cells; after thymectomy, clinical evaluation was good. Here, we describe this case with the aim of exploring and illustrating the relationship between MG and TC, and we discuss the possible links between the two entities.

\section{Materials and Methods}

The patient's information was obtained under signed consent, from the Ambulatory of Neuromuscular Disease of Santa Marcelina Hospital, São Paulo, Brazil, according to current ethical standards. The histological material was reviewed at the University of São Paulo, Department of Pathology.

\section{Case Report}

A 59-year-old Brazilian man, native and coming from São Paulo-SP, with a history of alcohol abuse and smoking, sought medical care in 2015 for progressive weakness, which had started 5 months previously. He presented with fatigable left eyelid ptosis, cervical and superior limb weakness, dysphagia, dyspnea, and dysarthria. He initially sought another hospital where he remained hospitalized to investigate and treat symptoms. With clinical suspicion of MG, he was put on prednisone and pyridostigmine, with partial improvement in symptoms. Later referred to our neurology medical outpatient clinic, patient was submitted to electroneuromyography, which depicted a decrement pattern at low rates of repetitive nerve stimulation. Blood test was positive for antiAChR antibodies. Thymic mass screening with chest computed tomography disclosed a small anterior mediastinal tumor, $26 \times 14 \mathrm{~mm}$ in diameter (-Fig. 1). Then patient was referred to thoracic surgery department, and video-assisted thymectomy was performed, $\sim 1$ year after symptom presentation. Pathological examination revealed squamous cell carcinoma of the thymus, G1 grade, with a lobular growth pattern and lymphocytes with a mature appearance (-Fig. 2). Resection margins were completely free of neoplasia. The tumor was stage I according to the Masaoka system ${ }^{13}$ (capsulated tumor with no microscopic capsular invasion) and TNM classification (T1 NO M0), with no lymph node or hematogenous metastasis. There was also no evidence of residual tumor after resection.

Since diagnosis, patient has been treated with pyridostigmine, oral steroids, and azathioprine, remaining in follow-up with oncology and neurology teams. After thymectomy, patient's medication doses were maintained for a period of approximately 1 year (prednisone $60 \mathrm{mg} /$ day, azathioprine $200 \mathrm{mg} / \mathrm{day}$, and pyridostigmine $90 \mathrm{mg} 4$ times daily), and then prednisone dosage was gradually decreased without recurrence of the symptoms. In last clinics consultation, he was on prednisone $5 \mathrm{mg} /$ day, azathioprine $200 \mathrm{mg} / \mathrm{day}$, and pyridostigmine $60 \mathrm{mg} 4$ times daily. His daily function remains good, with no signs of recurrence or metastasis.
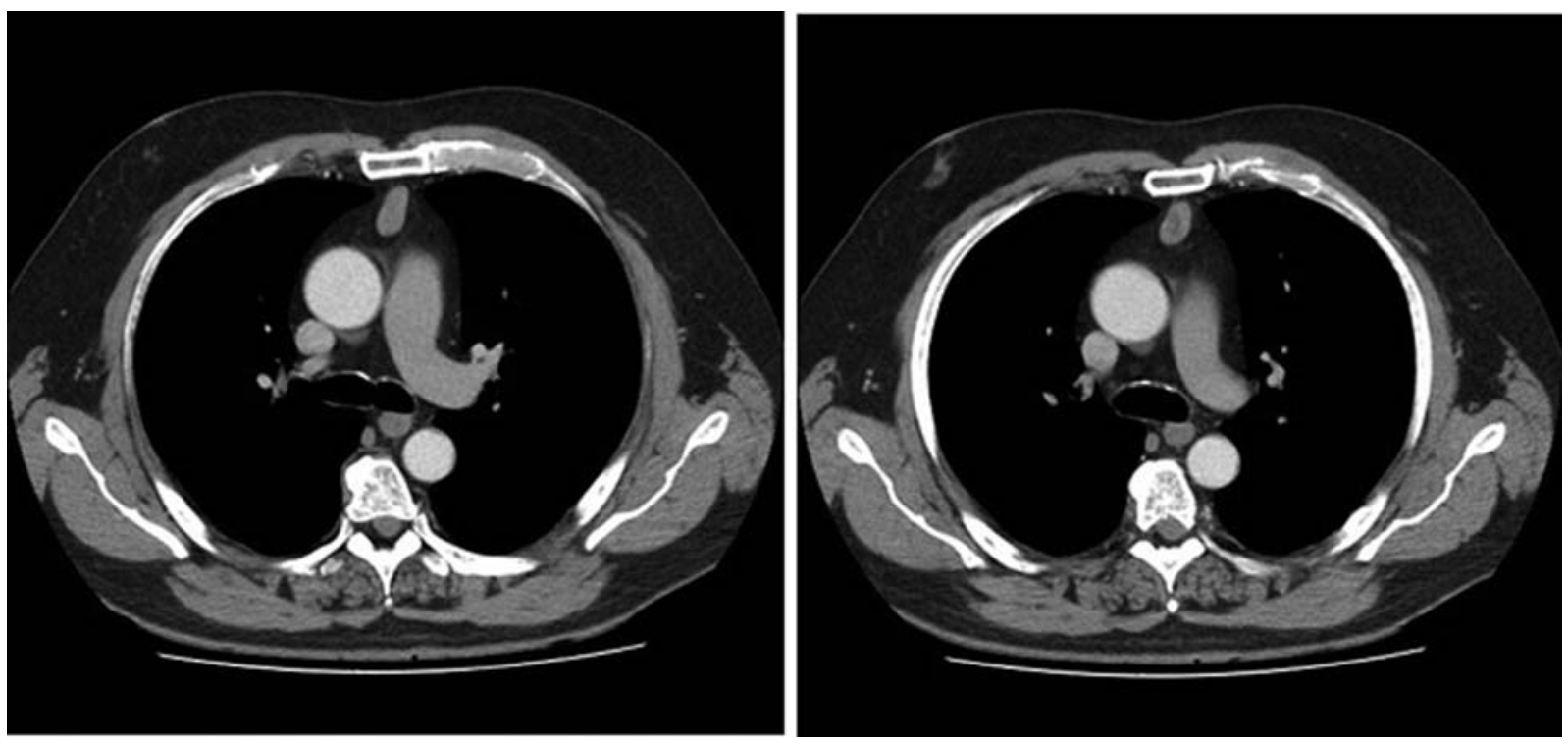

Fig. 1 Chest computed tomography. Anterior mediastinal tumor, $26 \times 14 \mathrm{~mm}$ in diameter, no apparent lymph node enlargement. 


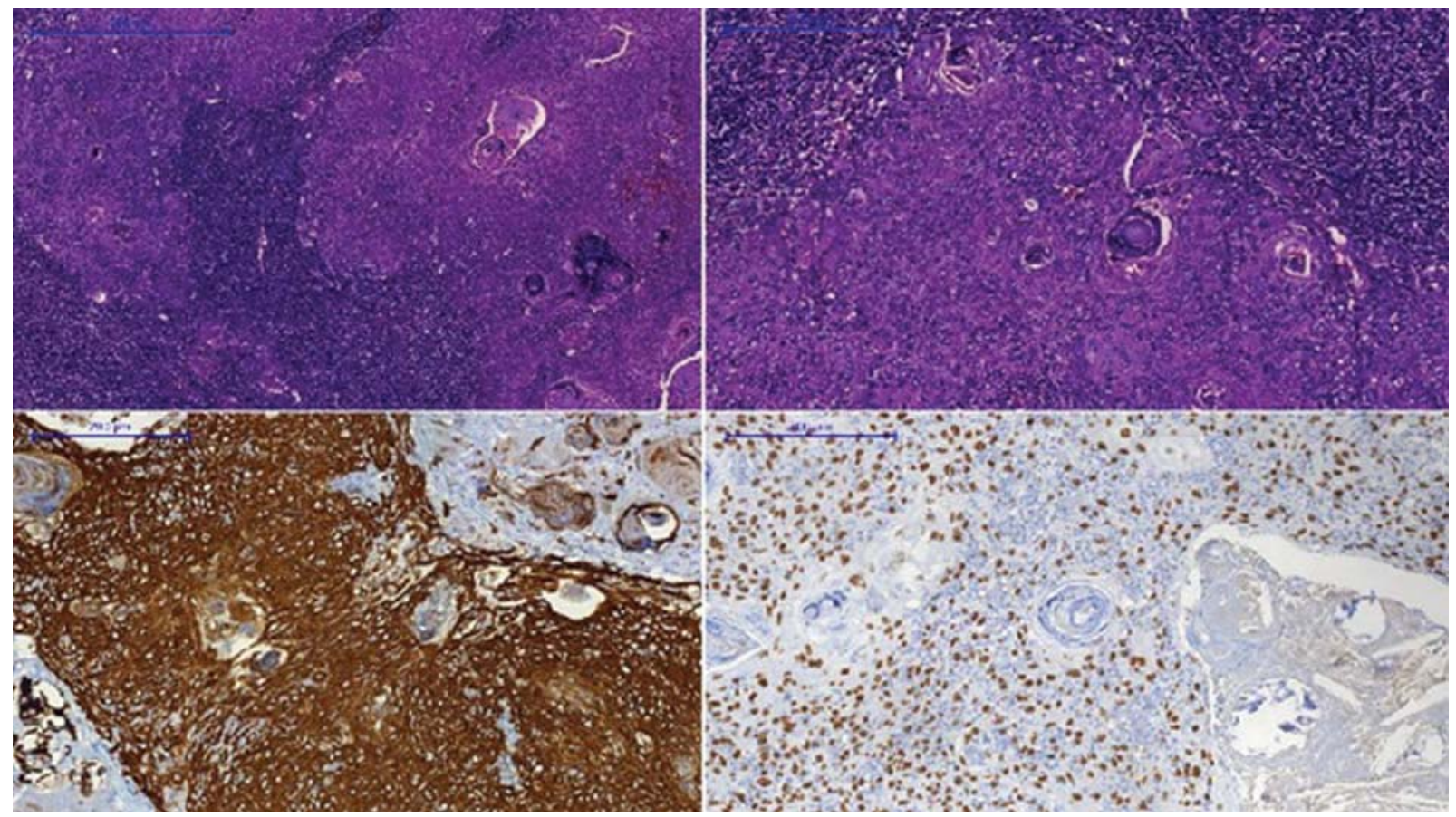

Fig. 2 Histopathological features of an epidermoid keratinizing squamous cell carcinoma, with a lobular growth pattern and mature lymphocytes. Hematoxylin staining.

\section{Discussion}

MG is a heterogeneous disease with several possible autoantigen targets at the neuromuscular junction, each theoretically resulting in a different pathogenesis. Some can be associated with thymic pathologies. The relationship between MG, antiAChR antibodies, and thymoma is well known, with 10 to $20 \%$ of patients with MG having an associated thymoma. Most paraneoplastic autoimmune diseases in other types of cancers are triggered by autoantigen epitopes in the tumor cells, which are also present in the target organ. Generally, a small minority of cancer cases develop paraneoplastic syndrome (usually $<5 \%$ ). However, higher incidence of paraneoplastic syndromes is seen in tumors originating from the thymus ( $50 \%)$, which might be related to additional immunologic features.

AChRs are expressed in the healthy thymus, in noninnervated thymic myoid cells, and thymic epithelial cells, ${ }^{14}$ which are needed to induce deletion of autoreactive $T$ cells against AChR. ${ }^{15}$ However, not all AChR-reactive cells are deleted, and some can include the normal $\mathrm{T}$ cell reserve, being kept inactive due to T cell anergy, "ignorance," or control in the extrathymic periphery by regulatory T cells. ${ }^{7}$ A breakdown of tolerance is likely in patients with MG, which would mainly be due to the abnormal expression of major histocompatibility complex class II and accessory molecules, changing the selection of molecules via apoptosis. Conversely, MG-related thymomas likely generate and export autoreactive $\mathrm{T}$ cells owing to the abnormal intratumorous microenvironment where thymocytes mature. ${ }^{15}$ In addition, such cases have defective expression of the autoimmune regulator, which normally drives "promiscuous" expression of peripheral tissue autoantigens (including the AChR $\alpha$-subunit in medullary thymic epithelial cells). ${ }^{15}$

For thymus squamous cell carcinoma, the theory about the emergence of MG is not well defined, given the rarity of the disease and small number of studies. The majority of studies on this subject are case descriptions, series of cases, or cohort of several thymic neoplasms that don't approach directly the association of squamous cell carcinoma and MG ( - Table $\mathbf{1}$ ).

Table 1 Database search results for the relationship between myasthenia gravis and thymic carcinoma. Search performed on September 15, 2019

\begin{tabular}{|l|l|l|l|}
\hline Database & Search strategy (descriptors) & Articles found & Articles included \\
\hline \multirow{3}{*}{ MEDLINE (PubMed) } & (myasthenia gravis) AND (thymic carcinoma) & 2,936 & 9 \\
\cline { 2 - 4 } & NOT (thymoma) & 17 & 0 \\
\hline \multirow{3}{*}{ oochrane Library } & (myasthenia gravis) AND (thymic carcinoma) & 3 & 1 \\
\cline { 2 - 4 } & NOT (thymoma) & 1 & 1 \\
\hline \multirow{2}{*}{ LILACS } & (myasthenia gravis) AND (thymic carcinoma) & 37 & 0 \\
\cline { 2 - 4 } & NOT (Thymoma) & 0 & 0 \\
\hline
\end{tabular}


One possible pathogenic mechanism is the development of TC inside a thymoma, with the remaining lymphoid cells being responsible for inducing antibody production, in a manner similar that of pure thymomas. When it arises from a thymoma, develops in the necrotic tissue ${ }^{8}$, more frequently inside a type B3 thymoma. ${ }^{12}$

In the present case, however, there was no evidence of any thymoma component. Our data seem reliable, as the tumor was quite small, with all margins free. It is possible that myasthenia in squamous cell carcinoma could have a more usual paraneoplastic mechanism, resembling other types of paraneoplastic syndromes. Then, the lower incidence of MG in patients with TC (compared with thymoma) could be justified by the lack of autoimmune facilitators present in thymoma patients, a kind of tumor that can have an incidence of paraneoplastic syndromes up to ten times the incidence of other tumors.

Several factors may predict the outcome of patients with TC: completeness of resection, Masaoka stage, histology, tumor size, and lymph node status. ${ }^{10}$ Although TC is aggressive, our patient has some good prognostic factors and is progressing well so far, from oncology and neurology perspectives. His MG symptoms are similar to those in other patients with anti-AChR and generalized weakness. Some authors report better prognosis of TC when it is associated with $\mathrm{MG},{ }^{10}$ which may be due to early diagnosis provided by the nonsilent manifestation. In addition, MG seems to be associated with more differentiated tumors, and the autoimmunity per se could have a protector role. However, it is a conflicting point, as other studies have shown that there is no difference in recurrence-free survival between patients with and without myasthenia. ${ }^{11}$

In addition to illustrating a very rare diagnosis of thymic malignancy with an even rarer association, this case report suggests TC might elicit anti-AChR MG, probably in a different way than by thymoma. A protective factor of MG for TC may still be speculated, although further studies are necessary for more robust evidence.

\section{Conflict of Interest}

None declared.

\section{Acknowledgments}

All authors have significantly contributed to this work, and the specific contributions are as follows:

1) Daniel Thomas Lopes: study concept, acquisition and analysis of data, literature review, and initial draft of the paper.

2) Samila Marissa Pinheiro Gomes: study concept, acquisition and analysis of data, literature review, and initial draft of the paper.

3) Fernando Pereira Frassetto: acquisition of data, literature review, and critical revision of manuscript for intellectual content.

4) Sheila Aparecida Siqueira: acquisition of data, literature review, and critical revision of manuscript for intellectual content.
5) Cristiane Martins Araújo Moreno: acquisition of data, literature review, and critical revision of manuscript for intellectual content.

6) Pedro Henrique Arruda Sampaio: acquisition of data, literature review, and critical revision of manuscript for intellectual content.

7) Maria Sheila Guimarães Rocha: acquisition of data, literature review, and critical revision of manuscript for intellectual content.

8) Eduardo de Paula Estephan: study concept, acquisition and analysis of data, literature review, and critical revision of manuscript for intellectual content.

\section{References}

1 Carr AS, Cardwell CR, Mccarron PO, Mcconville J. A Systematic Review of Population Based Epidemiological Studies in Myasthenia Gravis. Vol 10. 2010. Available at: http://www.biomedcentral. com/1471-2377/10/46. Accessed June 16, 2020

2 Berrih-Aknin S, Le Panse R. Myasthenia gravis: a comprehensive review of immune dysregulation and etiological mechanisms. J Autoimmun 2014;52:90-100

3 McMacken G, Abicht A, Evangelista T, Spendiff S, Lochmüller H. The increasing genetic and phenotypical diversity of congenital myasthenic syndromes. Neuropediatrics 2017;48(04):294-308

4 Abath Neto O, Heise CO, Moreno CA, et al. Nonlethal CHRNA1related congenital myasthenic syndrome with a homozygous null mutation. Can J Neurol Sci 2017;44(01):125-127

5 Sommer N, Harcourt GC, Willcox N, Beeson D, Newsom-Davis J. Acetylcholine receptor-reactive $\mathrm{T}$ lymphocytes from healthy subjects and myasthenia gravis patients. Neurology 1991;41(08): 1270-1276

6 Grativvol RS, Silva AM, Guedes BF, et al. Facial and bulbar muscle atrophy in acetylcholine receptor antibody-positive myasthenia gravis. Arq Neuropsiquiatr 2017;75(03):197-198

7 Khan MS, Habib A, Basit I. Unilateral-external ophthalmoplegia: a rare presentation of myasthenia gravis. J Coll Physicians Surg Pak 2016;26(11):142-143

8 Kurihara N, Saito $\mathrm{H}$, Nanjo $\mathrm{H}$, et al. Thymic carcinoma with myasthenia gravis: two case reports. Int J Surg Case Rep 2016; 27:110-112

9 Syrios J, Diamantis N, Fergadis E, et al. Advances in thymic carcinoma diagnosis and treatment: a review of literature. Med Oncol 2014;31(07):44. Doi: 10.1007/s12032-014-0044-2

$10 \mathrm{Li} \mathrm{W,} \mathrm{Miao} \mathrm{Z,} \mathrm{Liu} \mathrm{X,} \mathrm{et} \mathrm{al.} \mathrm{Thymic} \mathrm{carcinoma} \mathrm{patients} \mathrm{with}$ myasthenia gravis exhibit better prognoses. Int $\mathrm{J}$ Clin Oncol 2016;21(01):75-80

11 Nakajima J, Okumura M, Yano M, et al; Japanese Association for Research of Thymus. Myasthenia gravis with thymic epithelial tumour: a retrospective analysis of a Japanese database. Eur J Cardiothorac Surg 2016;49(05):1510-1515

12 Kim SH, Koh IS, Minn YK. Pathologic finding of thymic carcinoma accompanied by myasthenia gravis. J Clin Neurol 2015;11(04): 372-375

13 Kondo K. Tumor-node metastasis staging system for thymic epithelial tumors. J Thorac Oncol 2010;5(10, Suppl 4):S352-S356

14 Schluep M, Willcox N, Vincent A, Dhoot GK, Newsom-Davis J. Acetylcholine receptors in human thymic myoid cells in situ: an immunohistological study. Ann Neurol 1987;22(02):212-222

15 Marx A, Willcox N, Leite MI, et al. Thymoma and paraneoplastic myasthenia gravis. Autoimmunity 2010;43(5-6):413-427 